\title{
INSTRUMENTO PARA ENCAMINHAMENTO DE USUÁRIOS A SERVIÇOS SUBSTITUTIVOS DE SAÚDE MENTAL: RELATO DE EXPERIÊNCIA
}

INSTRUMENT FOR USERS 'REFERRAL TO REPLACING MENTAL HEALTH SERVICES: EXPERIENCE REPORT

Elisângela Seidel Maier', Amanda de Lemos Mello², Daiana Foggiato de Siqueira ${ }^{3}$, Marlene Gomes Terra ${ }^{4}$

RECEBIDO EM: 09/06/2017 / APROVADO EM: 01/11/2017

DOI: $10.5902 / 2317175827595$

\section{RESUMO}

Trata-se de um relato sobre a construção de um instrumento para encaminhamento do usuário com alta hospitalar de uma unidade de internação psicossocial à serviços substitutivos. Relato de Experiência: foi construído um instrumento com vistas a auxiliar no encaminhamento dos usuários de uma unidade de internação psicossocial à serviços substitutivos. Esse instrumento foi desenvolvido a fim de subsidiar o cuidado ampliado de acordo com a integralidade do usuário, bem como auxiliar na continuidade/adesão do cuidado à saúde. Conclusão: As contribuições para a saúde dos usuários foram na perspectiva de subsidiar o processo de alta e adesão dos usuários encaminhados da unidade psicossocial para os serviços substitutivos, bem como ofertar um mecanismo de co-responsabilização dos profissionais envolvidos na terapêutica dos usuários.

Palavras-chave: Alta do usuário; Encaminhamento; Serviços de saúde mental.

1 Terapeuta Ocupacional. Residente do Programa de Residência Multiprofissional Integrada em Saúde Mental da Universidade Federal de Santa Maria (UFSM). E-mail: elismaiers@gmail.com

2 Enfermeira. Mestra em Enfermagem. Doutoranda no Programa de Pós-GRaduação em Enfermagem da Universidade Federal de Santa Maria, Santa Maria, Rio Grande do Sul. E-mail: amandamello6@yahoo.com 3 Enfermeira. Mestra em Enfermagem. Doutoranda do Programa de Pós-Graduação em Enfermagem da UFSM. E-mail: daianasiqueira@gmail.com

4 Enfermeira. Doutora em Enfermagem. Docente do Departamento de Enfermagem da UFSM. E-mail: martesm@hotmail.com.br 
INSTRUMENTO PARA ENCAMINHAMENTO DE USUÁRIOS A SERVIÇOS SUBSTITUTIVOS DE SAÚDE MENTAL: RELATO DE EXPERIÊNCIA

\begin{abstract}
This is an account about the construction of an instrument for referral of the user with hospital discharge from a psychosocial hospitalization unit to substitutive services. Experience Report: An instrument was built to assist in the referral of users of a psychosocial care unit to substitutive services. It was developed in order to subsidize extended care according to the integrality of the user, as well as to assist in the continuity / adherence of health care. Conclusion: The contributions to the health of users were aimed at subsidizing the process of discharge and adherence of users referred from the psychosocial unit to substitutive services, as well as a mechanism of accountability of professionals involved in the treatment of users.
\end{abstract}

Keywords: Patient discharge; Referral; Mental health services

\title{
1 Introdução
}

O presente artigo trata sobre a continuação/adesão do tratamento pós -alta de uma unidade psicossocial visando o processo de vinculação do usuário à serviços substitutivos. Essa temática emerge a partir da Reforma Psiquiátrica brasileira com a Lei 10.216, de 06 de abril de 2001 a fim de revogar o modelo de assistência voltado ao isolamento. Com a promulgação dessa Lei e a reorientação dos serviços, surge a necessidade de uma rede de atenção integral em saúde mental a fim de diminuir o número de internações e reinternações psiquiátricas. Tem-se, assim, a possibilidade de um suporte ao usuário em base territorial e nos serviços substitutivos (SIQUEIRA et al, 2015).

Os serviços substitutivos, como os Centros de Atenção Psicossocial (CAPS), são configurados como os de saúde que prestam atendimento extra hospitalar aos usuários de saúde mental. Os CAPS podem atender os usuários a partir do encaminhamento pelo sistema de referência e contrarreferência com vistas a diminuir a ocorrência de (re)internações hospitalares (AZEVEDO et al., 2011).

O Sistema de Referência e Contrarreferência é uma configuração dos serviços de saúde em redes, organizado por critérios, fluxos e pactuações de funcionamento, para assegurar a atenção integral aos usuários. Esse sistema prevê o atendimento em diferentes níveis de complexidade, permitindo o encaminhamento resolutivo entre os serviços de saúde (COSTA et al., 2013).

Para que esse processo de encaminhamento ocorra de modo efetivo, faz-se necessário que os profissionais envolvidos na atenção à saúde compreendam para além dos aspectos clínicos, como exemplo, observar os fatores demográficos, raça, gênero, estado civil, suporte familiar e apoio da rede psicossocial das pessoas. Além disso, é necessário que eles possam compreender as condições físicas e mentais, os motivos tanto da atual internação como as anteriores e, também da proposta terapêutica (NAKANISHI et al., 2010).

Evidências apontam para a necessidade de se trabalhar questões relativas ao encaminhamento dos usuários de saúde mental, questões estas que apresentam dificuldades na sua efetivação. Essas dificuldades podem estar re- 
lacionadas, entre outros motivos, ao excesso de demanda, falta de tempo, ausência de comunicação e falhas no planejamento pós-alta hospitalar (NAKANISHI et al., 2010; BATSCHA et al., 2011). Além disso, a realização deficiente da contrarreferência apontam falhas no sistema de saúde, que afetam a integralidade no atendimento dos usuários (MENOZZI, 2013).

Assim, justifica-se este estudo por possibilitar a criação de um instrumento com foco em auxiliar na continuidade do cuidado de atenção em saúde mental e facilitar o registro/documentação da atenção à saúde no encaminhamento dos usuários internados em uma unidade psicossocial à serviços substitutivos. Além disso, tem-se como objetivo relatar a construção de um instrumento para encaminhamento do usuário com alta hospitalar de uma unidade psicossocial à serviços substitutivos.

\section{Relato de experiência}

\subsection{Contextualização do cenário}

Este relato é oriundo do projeto de pesquisa intitulado "Alta hospitalar com encaminhamento ao centro de atenção psicossocial: percepção de usuários e trabalhadores da saúde", cujo objetivo geral foi compreender a alta hospitalar na perspectiva de trabalhadores dos serviços e usuários egressos de uma unidade de internação psiquiátrica de um hospital de ensino. Essa pesquisa foi desenvolvida em uma unidade de internação psicossocial de um Hospital de Ensino localizado em um município no interior do estado do Rio Grande do Sul, Brasil. $O$ instrumento foi criado durante a realização desta pesquisa, no período de março de 2016 a janeiro de 2017 posterior a Aprovação do Comitê de Ética sob o Parecer $N^{\circ} 1.538 .373$ e CAAE No 5534116.4.0000.5346, que segue a Resolução $N^{\circ}$ 466/2012 do Conselho Nacional de Saúde.

A produção de dados da pesquisa supracitada foi realizada por meio de entrevistas semiestruturadas, com a participação de oito profissionais que atuavam na unidade psicossocial do referido Hospital de Ensino, 10 profissionais de um serviço substitutivo (CAPS II) e 10 usuários que passaram por uma internação psiquiátrica e foram encaminhados ao serviço substitutivo no ano de 2015.

Tratando dos resultados desta pesquisa, estes mostraram a percepção de trabalhadores e usuários sobre o compartilhamento do cuidado na alta com encaminhamento ao CAPS. Também foi possível conhecer que esse processo de continuidade da atenção à saúde, na maioria das vezes, ocorre por meio do protagonismo da equipe médica, via ofícios com dados gerais sobre a clínica do usuário. Além disso, as equipes dos serviços substitutivos, por vezes, não conhecem o usuário, seu histórico e possível proposta terapêutica, dificultando a adesão e o vínculo do mesmo nestes locais.

Evidenciou, ainda, que alguns fatores podem vir a interferir na qualidade da continuação da atenção à saúde, como: acolhimento ofertado na rede que dá o suporte na pré-alta (profissionais de referência); conhecimento e 
INSTRUMENTO PARA ENCAMINHAMENTO DE USUÁRIOS A SERVIÇOS SUBSTITUTIVOS DE SAÚDE MENTAL: RELATO DE EXPERIÊNCIA

aceitação do usuário de seu diagnóstico e possibilidade de tratamento terapêutico; e, também os que dizem respeito ao envolvimento da família no cuidado desse usuário. Esses fatores foram capazes de traduzir a situação real ou potencial do processo de encaminhamento dos usuários internados em uma Unidade de Internação Psicossocial (UIP) aos serviços substitutivos. E, que, a partir disso foram a base para orientar ações de intervenção, bem como, as formas de auxiliar no encaminhamento desses usuários.

A partir das evidências na produção das informações e das necessidades dos serviços encontradas no banco de dados da pesquisa, foi discutido entre a equipe de residentes de saúde mental e trabalhadores da UIP a possibilidade de construir um instrumento. Este poderia ser capaz de favorecer qualidade do encaminhamento da assistência no pós-alta hospitalar, possibilitando uma maneira mais adequada ao processo de vinculação do usuário a serviços substitutivos.

$O$ instrumento busca padronizar e normatizar uma linha de cuidado, pensada em equipe, com vistas a garantir a integralidade e continuidade do cuidado ao usuário de saúde mental, no período de internação, de alta hospitalar, do vínculo com a rede e de retorno ao domicílio. Assim, essa ferramenta foi construída de forma coletiva pelos residentes do Programa de Residência Multiprofissional e Integrada em Saúde Mental, com a articulação da Coordenadoria Regional de Saúde, a qual o cenário do estudo de campo estava vinculado.

A rede de saúde mental do município estudado é constituída por serviços de saúde voltados às pessoas com transtorno mental e aos usuários de drogas, como: dois CAPS Ad, um CAPS infantil, um CAPS II, um Ambulatório de Saúde Mental e uma unidade de internação psicossocial em um Hospital de Ensino de grande porte para atendimento a adultos.

Os encaminhamentos dos usuários internados na UIP é uma ação realizada pela equipe de residentes multiprofissionais por intermédio de contatos prévios com a rede via telefone. É dessa maneira que são firmados os acordos com o serviço e no momento da alta é orientando o familiar e o usuário a procurar o CAPS. Diante do processo de trabalho na UIP, percebeu-se a necessidade de construir uma ferramenta de comunicação capaz de favorecer uma melhor qualidade no encaminhamento (pós-alta) dos usuários internados na referida unidade de internação ao CAPS, possibilitando uma maneira mais adequada ao processo de vinculação do usuário ao serviço.

No processo da alta hospitalar dá-se transferência do cuidado. Esta é realizada por meio de orientações ao usuário e seu familiar/cuidador em relação à continuidade do tratamento, empoderando quanto à autonomia do sujeito de modo a propiciar o autocuidado. Por meio da referência e contrarreferência, deve ser realizado o cuidado continuado em todos os pontos de atenção à saúde.

Nesse sentido, tem-se o profissional de referência nos serviços de saúde mental do município, em que pode ser considerado como uma possibilidade para potencializar reformulações na realidade de saúde mental. Esta forma de trabalhar visa valorizar a construção do vínculo com o usuário como instru- 
mento essencial do trabalho, a partir do acompanhamento do projeto terapêutico e dos objetivos traçados junto ao usuário e seus familiares.

Em relação ao CAPS II, este atende a demanda de transtornos mentais advindos de internação da UIP ou encaminhados de outros serviços, atende em média 800 usuários em situações moderadas e graves.

\subsection{A construção do instrumento}

O instrumento desenvolvido para operacionalizar o encaminhamento dos usuários vinculados à uma UIP para serviços substitutivos possui três eixos geradores. Esses correspondem à atenção ofertada aos usuários, sendo: dados gerais da internação; aspectos gerais do serviço que o usuário será encaminhado; e informações referentes a seu tratamento de acordo com dados psicossociais (ANEXO 1).

Para que o usuário acesse os serviços substitutivos, faz-se necessário fornecer os seguintes dados para o encaminhamentos para serviços substitutivos em saúde mental: nome, endereço, contato e Coordenadoria Regional de Saúde do serviço para onde está sendo encaminhado, data que o profissional responsável entrou em contato com o serviço para passar informações da alta, data e horário do agendamento do usuário no serviço após a alta, profissional responsável pelo acolhimento do usuário no serviço substituto e sua função.

As informações sobre o período de internação são relevantes ao tratamento pós alta do usuário, sendo Informações do Tratamento: Aspectos Psicossociais, em que constam vínculo com a equipe, participação em atividades na unidade (descrição da atividade, grau de envolvimento), atendimentos realizados, participação da família (quem visitou e/ou participou de orientação ou de grupos de familiares; observação da equipe quanto a dinâmica familiar), comportamento durante a internação e observações e recomendações terapêuticas/necessidades avaliadas.

Por fim, identifica-se o nome do responsável pelas informações, a função e a formação e a data do preenchimento. Deste modo, o serviço substitutivo identifica o profissional que foi responsável pelo encaminhamento, podendo a qualquer momento realizar contato para sanar dúvidas sobre o usuário e sua internação na unidade psiquiátrica.

Ressalta-se que as informações sobre o tratamento clínico, como medicações e doses utilizadas durante a internação; reações adversas; evolução do tratamento e grau de remissão da sintomatologia; medicação prescrita para o pós-alta e doses; recomendações; nome do médico responsável pelas informações e registro no Conselho Regional de Medicina, estão descritos no encaminhamento efetuado pelo médico. Sendo que este já existe e é realizado na unidade pela equipe médica. 
INSTRUMENTO PARA ENCAMINHAMENTO DE USUÁRIOS A SERVIÇOS SUBSTITUTIVOS DE SAÚDE MENTAL: RELATO DE EXPERIÊNCIA

\section{Discussão}

O sistema de referência e contrarreferência da rede de saúde mental do município estudado, conforme apontam autores, apresenta-se pouco efetivo. Dentre os fatores que interferem na assistência em saúde mental, encontra-se falhas na circulação de informações, desconhecimento pelos próprios profissionais em relação aos serviços que constituem a rede e aos encaminhamentos após a internação hospitalar. Dessa maneira, a rede encontra-se fragmentada, tendo, por vezes, a descontinuidade nos tratamentos (ROSA et al., 2013)

A rede de atenção psicossocial funciona com base no trabalho das equipes de saúde, essas podem articular de uma unidade básica para um CAPS, ou desta para um hospital. Isto significa que em cada caso, os caminhos a serem percorridos são acionados e traçados nas demandas dos usuários e nos recursos disponíveis para o seu cuidado (QUINDERÉ, JORGE \& FRANCO, 2014).

A responsabilidade compartilhada, entre os profissionais da equipe interdisciplinar, do cuidado com os usuários é uma das principais atribuições dos serviços de saúde mental na efetivação da integralidade da assistência (ANDRADE \& BOSI, 2015). Nessa perspectiva, reconhece-se o importante papel dos profissionais de saúde atuantes em UIPs, pois vivenciam mudanças estruturais, operacionais e organizacionais, além da alteração no fluxo das práticas a partir da adoção de outros serviços (PESSOA et al., 2016).

No cotidiano desses serviços de saúde, percebe-se que os encaminhamentos dependem, por vezes, de planejamento da continuidade da assistência pelos profissionais envolvidos no cuidado ao usuário. O planejamento sistemático da alta hospitalar pode contribuir na diminuição do quantitativo de reinternações, assim como evitar o surgimento de complicações durante o tratamento do usuário (MATTEl et al., 2014).

Esse planejamento pode ser operacionalizado por meio da construção de instrumentos, considerados importantes nas ações de saúde, com vistas a otimizar a assistência e facilitar o fluxo da rede (MATTEl et al., 2014). Além disso, a elaboração de instrumentos que direcionem o cuidado e auxiliem o usuário na continuidade do tratamento, tendem a proporcionar um cuidado ampliado (HAYDEN et al., 2010). A construção de instrumentos pode facilitar a comunicação na rede e acompanhamento do progresso no tratamento do usuário. Também contempla ações intersetoriais, em que o vínculo entre o usuário e os serviços substitutivos, podem ser estabelecidos por meio de referência e contrarreferência. Assim, esses mecanismos podem garantir a continuidade do cuidado da saúde mental (THIEME, R.D., et al., 2014).

Nesse sentido, de acordo com a Reforma Psiquiátrica, o planejamento da assistência necessita abordar para além dos aspectos clínicos. O modelo de atenção psicossocial implica em ações que incluem o reposicionamento dos profissionais da saúde na assistência para atingir a integralidade do cuidado em saúde mental. Essa perspectiva pressupõe um olhar para além da doença, em que en- 
globe as necessidades psicossociais dos usuários. A partir da experiência descrita da construção do instrumento que visa facilitar no encaminhamento dos usuários egressos de uma UIP para serviços substitutivos, percebeu-se a importância de elementos que perpassam o olhar clínico centrado na doença. É necessário observar aspectos subjetivos que implicam no tratamento, como vínculo com a equipe, participação da família e comportamento durante a internação.

O vínculo estabelecido entre os profissionais e os usuários podem ser considerados como uma estratégia voltada à promoção da integralidade e a singularidade do cuidado em saúde. Esse vínculo pode ser de relevância para possibilitar um viver saudável aos indivíduos, famílias e comunidade, de forma singular e contextualizada, por meio do reconhecimento da prática e potencialização dos recursos e competências de cada indivíduo (ILHA et al., 2014).

Nesse ínterim, a participação da família influencia na atenção psicossocial, pois o usuário que estabelece vínculo com ela durante o processo de alta hospitalar com encaminhamento a serviços substitutivos tem maior adesão e aproveitamento ao tratamento. Além disso, o apoio da família estimula o usuário a se manter na rede de saúde mental (ROSA, et al., 2013).

A internação em saúde mental, após Reforma Psiquiátrica, é baseada em atividades humanas afetivas e interdisciplinares estando continuamente em processo de transformação dos conceitos relacionados ao universo da saúde mental. Sendo assim, o tratamento ao usuário é estabelecido de modo a respeitar suas subjetividades, com isso, o comportamento do usuário pode ser favorável a aceitação/adesão ao tratamento. O apoio para a aceitação ao tratamento por parte do usuário, pode ocorrer tanto durante o processo de internação, quanto no encaminhamento aos serviços substitutivos. Mas para isso, é importante que sejam proporcionados espaços para discussões e formação de vínculos dos usuários com os profissionais, usuários e familiares (MELLO \& SCHNEIDER, 2011).

\section{Considerações finais}

O presente estudo permitiu a construção de um instrumento com vistas a subsidiar a alta hospitalar na lógica de interlocução entre os serviços de internação psicossocial hospitalar aos serviços substitutivos. Pode se constituir como um instrumento facilitador na identificação das principais informações e demandas do usuário, bem como ofertar um mecanismo de corresponsabilização dos profissionais envolvidos na terapêutica dos usuários.

A elaboração desse instrumento poderá subsidiar a formalização da alta hospitalar a partir de um planejamento da alta corresponsável. Além disso, poderá promover a articulação dos diferentes pontos de atenção em saúde e o cuidado integral ao indivíduo, com vistas a favorecer a referência e contrarreferência. Como limitação deste estudo, tem-se que o instrumento proposto não foi aplicado e validado na prática clínica. Assim, sugere-se sua validação de conteúdo e clínica com adequação para a realidade e especificidade de cada instituição. 
INSTRUMENTO PARA ENCAMINHAMENTO DE USUÁRIOS A SERVIÇOS SUBSTITUTIVOS DE SAÚDE MENTAL: RELATO DE EXPERIÊNCIA

\section{Referências}

SIQUEIRA, D.F., et al. Reinserção social do indivíduo dependente de crack: ações desenvolvidas pela família. Revista Texto Contexto Enfermagem, v. 24, n.2, p. 548-53, 2015.

AZEVEDO, D.M., et al. Avaliação da assistência em saúde num centro de atenção psicossocial na perspectiva dos profissionais. Revista Brasileira de Pesquisa em Saúde, v. 16, n. 2, p. 109-16, 2011.

COSTA, S.M., et al. Referência e contrarreferência na saúde da família: percepção dos profissionais de saúde. Revista de Atenção Primária à Saúde, v. 16, n.3, p. 287-93, 2013.

NAKANISHI, M., et al. Development of a clinical pathway for long-term inpatients with schizophrenia. Psychiatry Clinical Neurosciences, v. 64, n. 1, p. 99-103, 2010.

BATSCHA, C., et al. The effect of an inpatient transition intervention on attendance at the first appointment post discharge from a psychiatric hospitalization. J Am Psychiatr Nurses Assoc, v. 17, n. 5, p. 330-8, 2011.

MENOZZI, K. O sistema de referência e contra-referência no contexto da equipe multiprofissional de saúde. Universidade Estadual Paulista, Faculdade de Medicina de Botucatu. Botucatu: [s.n.], 2013.

ROSA, E.M.K., et al. Reconhecimento do fluxo dos usuários de saúde mental do município de Santa Maria. Revista Saúde, v. 39, n. 2, p. 8998, 2013.

QUINDERÉ, P.H.D., JORGE, M.S.B. \& FRANCO, T.B. Rede de Atenção Psicossocial: qual o lugar da saúde mental? Physis Revista de Saúde Coletiva, v. 24, n. 1, p. 253-71, 2014.

ANDRADE, A.B. \& BOSI, M.L.M. Qualidade do cuidado em dois centros de atenção psicossocial sob o olhar de usuários. Revista Saúde e Sociedade, v. 24, n.3, p. 887-900, 2015.

PESSOA, J.M., et al. Política de saúde mental no hospital psiquiátrico: desafios e perspectivas. Revista de Enfermagem Escola Anna Nery, v. 20, n. 1, p. 83-9, 2016.

MATTEI, A.T., et al. Elaboração de protocolos para a alta hospitalar de pacientes hipertensos e diabéticos: relato de experiência. Revista Ciência Cuidado \& Saúde, v. 13, n. 1, p. 160-65, 2014.

HAYDEN, B.B., et al. The implementation of A translational study involving a primary care based behavioral program to improve blood pressure control: The HTN-IMPROVE study protocol. Bosworth et al. Implementation Science, v. 5, n. 54, s/p., 2010.

THIEME, R.D., et al. Elaboração e implantação de protocolo de alta responsável para idosos com doenças crônicas hospitalizados e com necessidades alimentares especiais. Demetra: alimentação, nutrição \& saúde, v. 9, n. 1, p. 269-86, 2014.

ILHA, S., et al. Vínculo Profissional-Usuário Em Uma Equipe Da Estratégia Saúde Da Família. Revista Ciência Cuidado \& Saúde, v.13, n.3, p. 556-62, 2014.

MELLO, R.M. \& SCHNEIDER, J.F. A família e a internação psiquiátrica em hospital geral. Revista Gaúcha de Enfermagem, v. 32, n. 2, p. 226-33, 2011.

ANEXO 1: Instrumento para auxiliar no encaminhamento dos usuários de uma unidade de internação psicossocial à serviços substitutivos, Santa Maria, 2017. 